\title{
POBLACIONES DE LOMBRICES BAJO SEIS ESTRATEGIAS DE MANEJO DE MALEZAS EN UNA PLANTACIÓN DE BANANO ${ }^{1}$
}

\author{
Renán Agüero ${ }^{2}$, Silvia Rojas ${ }^{2}$, Leonardo Pérez ${ }^{3}$
}

\begin{abstract}
RESUMEN
Poblaciones de lombrices bajo seis estrategias de manejo de malezas en una plantación de banano. En la finca bananera San Pablo, ubicada en la Zona Atlántica, se establecieron seis tratamientos de manejo de malezas, para evaluar su efecto sobre el número de lombrices. Los tratamientos fueron: un testigo con libre crecimiento de las malezas, chapeas mensuales, aplicación de glifosato (Ranger Plus-680 $\mathrm{g} / \mathrm{kg}$; al $0,687 \% \mathrm{p} / \mathrm{v}$ ) cada dos meses, aplicación de paraquat (Gramoxone-200 g/l; al 0,75\% v/v) cada mes, aplicación de glufosinato (Finale-150 g/l; al 1\% v/v) cada dos meses y un tratamiento en el que se realiza un combate racional con herbicida y machete, sólo en las rodajas. Cada tratamiento constó de tres repeticiones, y en cada una se tomaron cuatro muestras de suelo de $15625 \mathrm{~cm}^{3}$ y se contaron las lombrices presentes. Las muestras se tomaron en diferentes microhabitats: bajo cobertura espontánea de Paspalum conjugatum o Panicum zizanoides, en suelo desprovisto de vegetación, y bajo hojarasca de banano en descomposición. Se observó que los diferentes manejos de malezas no afectaron la cantidad de lombrices, pero sí se determinó diferencias en la cantidad de lombrices entre los distinos hábitats. Una mayor población de lombrices fue encontrada en sitios bajo hojarasca de banano en descomposición y bajo Paspalum conjugatum y una menor población de estos organismos bajo Panicum zizanoides y en suelo desprovisto de vegetación.
\end{abstract}

\begin{abstract}
Earthworm populations under six weed control strategies in a banana plantation. Six weed management strategies were established in with banana plantation; in San Pablo, a farm located in the Atlantic region of Costa Rica. Since then, earthworm counts have been performed at regular intervals. Weed management strategies consisted of: 1unweeded control. 2- Hand weeded control at monthly intervals. 3- Glyphosate (Ranger Plus-680 g/kg; at $0.687 \%$ w/v) applications at eight week intervals. 4- Paraquat (Gramoxone-200 g/l, at $0.75 \% \mathrm{v} / \mathrm{v}$ ) at monthly intervals. 5Glufosinate (Finale-150 g/l, at 1\% v/v) applications at eight week intervals. 6- Monitored treatment, depending on detected weeds, only in the area of maximum weed-crop interference. Earthworms were counted, at each sampling time, from four $15625 \mathrm{~cm}^{3}$ soil samples of each plot. Samples were taken from different microhabitats, including soil covered with Paspalum conjugatum; soil covered with Panicum zizanoides; soil covered with banana residues (leaves, pseudo-stems) and bare soil. Soil samples from sites covered with banana residues or Paspalum conjugatum showed the highest earthworm counts, followed by soil covered with Panicum zizanoides and bare soil. A direct effect of weed management strategies on earthworm number has not been detected.
\end{abstract}

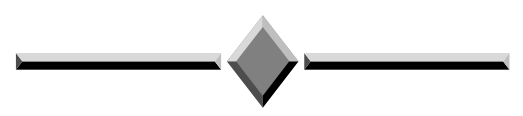

\section{INTRODUCCIÓN}

En los últimos años se ha venido desarrollando una conciencia ambientalista y se ha generalizado la creen- cia de que la mayoría de los manejos que se dan en plantaciones agrícolas afectan la calidad del medio, la cual puede ser medida a través de poblaciones de lombrices.

\footnotetext{
1 Recibido para publicación el 2 de octubre del 2001. Proyecto de investigación № 813-A1-802, inscrito en la Vicerrectoría de Investigación de la Universidad de Costa Rica.

2 Laboratorio de malezas, Centro de Investigaciones en Protección de Cultivos (CIPROC), Universidad de Costa Rica (UCR). E-mail: raguero@cariari.ucr.ac.cr.

3 Centro de Investigaciones Agrícolas, CORBANA, La Rita de Guápiles, Limón, Costa Rica.
} 
Las lombrices de tierra son anélidos oligoquetos terrestres y uno de los componentes más importantes de la fauna del suelo (León et al. 1988). Viven en túneles estrechos, profundos, oscuros y largos bajo la superficie (Day 1996). Se alimentan de materia orgánica en distintos grados de descomposición, que proviene de múltiples fuentes de desechos (Edward 1986, citado por León et al. 1988). Estos organismos ingieren partículas minerales, materia orgánica proveniente de plantas y animales muertos, y microorganismos (Donker et al. 1993).

Las lombrices contribuyen a la fertilidad del suelo al mejorar sus propiedades físicas, químicas y biológicas (Fraile 1986b). Sus excrementos son depositados en la superficie y son ricos en nutrientes, que son utilizados como alimento por otros animales y microorganismos. Este material orgánico es luego degradado por microorganismos del suelo, liberando nutrientes que son luego absorbidos por las plantas (Day 1996). Construyen galerías dentro del suelo que proporcionan mayor aireación, favorecen la infiltración del agua y la permeabilidad. Estas modificaciones del suelo, a su vez, producen cambios que benefician a otras especies animales y vegetales que habitan en él (Lee 1985, citado por León et al. 1988).

Estos organismos juegan un papel fundamental en el ecosistema del suelo al reciclar componentes químicos como por ejemplo metales pesados (Donker et al. 1993); también distribuyen materia orgánica y nitrógeno en las capas profundas y remontan materiales calcáreos del horizonte C a la superficie (Fraile 1986a).

Las lombrices de tierra podrían utilizarse como controladores de la contaminación orgánica y en la producción de proteína (Fraile 1986a). Estos organismos se consideran como el único bioindicador fisiológico, a nivel práctico, disponible en el suelo; de su bioacumulación se puede estimar el nivel de contaminación del ecosistema, gracias a su función clave y su fácil seguimiento (Donker et al. 1993).

Debido a la importancia y a la polémica actual alrededor de la conservación del suelo, se creyó conveniente publicar la información acumulada en este estudio; sin embargo, el mismo se continuará por un período de tres años, al final del cual se espera poner a disposición otros detalles que se hayan esclarecido.

El objetivo de la presente investigación fue determinar si el número de lombrices en una plantación de banano se reduce al aplicar en ella diferentes manejos de malezas, así como determinar los factores que afectan su población dentro de la plantación.

\section{MATERIALES Y MÉTODOS}

El estudio se inició el 17 de noviembre del 2000, en la finca San Pablo, de la Corporación Bananera Nacional (CORBANA), ubicada en 28 Millas de Limón, Costa Rica. Esta región se clasifica como bosque tropical húmedo, con una precipitación anual que fluctúa entre los 3500 y $4000 \mathrm{~mm}$. La temperatura promedio anual es de $25^{\circ} \mathrm{C}$ y la humedad relativa promedio es de $85 \%$.

En un sector de la plantación que fue sembrado con banano cv. Gran Enano (Musa AAA) hace 10 años, se establecieron seis tratamientos correspondientes a estrategias de manejo de malezas contrastantes. Se utilizó un diseño de bloques completos al azar con tres repeticiones. Cada parcela fue de $400 \mathrm{~m}^{2}$ (20x20 m). Los tratamientos fueron:

1: Testigo con libre crecimiento de malezas

2: Combate de malezas con chapea cada mes

3: Combate de malezas cada ocho semanas con herbicida post-emergente glifosato (Ranger Plus al 0,687\% $\mathrm{p} / \mathrm{v})$.

4: Combate de malezas cada cuatro o cinco semanas con herbicida post-emergente paraquat (Gramoxone) al $0,75 \% \mathrm{v} / \mathrm{v}$ más pega $(\mathrm{WK})$ al $0,5 \% \mathrm{v} / \mathrm{v}$.

5: Combate de malezas cada ocho semanas con herbicida post-emergente glufosinato (Finale) al $1 \% \mathrm{v} / \mathrm{v}$.

6: Combate mixto racional con glifosato, glufosinato y machete, en la rodaja, con base en la presencia de malezas.

En cada parcela experimental de cada uno de los seis tratamientos se tomó al azar dos muestras de suelo con cobertura espontánea y dos muestras de suelo bajo hojarasca de banano en descomposición. El término cobertura espontánea se utilizó para referirse a los muestreos que no son bajo hojarasca, es decir puede incluir Paspalum, Panicum y suelo descubierto. El número de muestras y la procedencia (número de parcela) es distinto para cada hábitat bajo lo que se denomina como cobertura espontánea, ya que esto depende de lo que se encontrara en cada parcela. Cada muestra consistió en un cubo de $15625 \mathrm{~cm}^{3}\left(0,016 \mathrm{~m}^{3}\right)$; en los cuales se recogieron manualmente las lombrices presentes y se contaron. Los muestreos se realizaron cada dos meses.

\section{Análisis de datos}

El anális estadístico se realizó mediante análisis de varianza y las medias se separaron por pruebas de rangos múltiples. 
Cuadro 1. Número de lombrices por $\mathrm{m}^{3}$ bajo cobertura espotánea en los diferentes tratamientos de manejo de malezas. Finca San Pablo.

\begin{tabular}{lrrrrrr}
\hline & \multicolumn{6}{c}{ Tratamientos } \\
\cline { 2 - 7 } Muestreo & Testigo & Machete & Glisofato & Paraquat & Glusifonato & Racional \\
\hline $17 / 11 / 00$ & $250 \mathrm{a}$ & $333 \mathrm{a}$ & $292 \mathrm{a}$ & $146 \mathrm{a}$ & $312 \mathrm{a}$ & $417 \mathrm{a}$ \\
$08 / 03 / 01$ & $552 \mathrm{a}$ & $250 \mathrm{a}$ & $271 \mathrm{a}$ & $229 \mathrm{a}$ & $239 \mathrm{a}$ & $625 \mathrm{a}$ \\
$19 / 05 / 01$ & $292 \mathrm{a}$ & $198 \mathrm{a}$ & $125 \mathrm{a}$ & $114 \mathrm{a}$ & $83 \mathrm{a}$ & $333 \mathrm{a}$ \\
$10 / 08 / 01$ & $489 \mathrm{a}$ & $396 \mathrm{a}$ & $167 \mathrm{a}$ & $364 \mathrm{a}$ & $229 \mathrm{a}$ & $437 \mathrm{a}$ \\
$10 / 10 / 01$ & $489 \mathrm{a}$ & $396 \mathrm{a}$ & $292 \mathrm{a}$ & $302 \mathrm{a}$ & $312 \mathrm{a}$ & $375 \mathrm{a}$ \\
\hline
\end{tabular}

Medias con la misma letra de una fila, no difieren según la prueba de DMS protegida de Fisher, al 0,05 de probabilidad

\section{RESULTADOS Y DISCUSIÓN}

En los cinco muestreos realizados (Cuadros 1 y 2) no se encontró diferencia en el número de lombrices entre los distintos tratamientos de manejo de malezas. Poter et al. (1990) y, Bauer y Römbke (1997), estudiaron la toxicidad que provocan distintos plaguicidas a las lombrices y no encontraron efectos en sus poblaciones al utilizar herbicidas. Según Lee (1985) citado por Potter et al. (1990), ninguno de los 33 herbicidas de los que se tuvo información disponible, empleados en las dosis recomendadas, resultaron tóxicos para las lombrices ni se acumulan en sus tejidos.

Cuando se analizó el número de lombrices bajo cobertura espontánea y bajo hojarasca de banano, tomando los tratamientos como repeticiones (Cuadro 3 ) se encontró que la hojarasca de banano promovía una mayor población de lombrices en los primeros $25 \mathrm{~cm}$ de profundidad del suelo, resultando que se repitió en todos los muestreos. Esto pudo deberse a un factor humedad. Fraile (1986b) indica que en los períodos secos, las lombrices se vuelven inactivas y se hunden profundamente en el suelo. La humedad bajo la hojarasca se mantiene bastante alta, incluso fue común encontrar varias lombrices en la superficie del suelo. Day (1996), menciona que las lombrices salen de sus túneles cuando llueve, porque es más fácil su movimiento en la superficie mojada.

En el Cuadro 4 se agruparon los datos para el análisis, de acuerdo a cuatro hábitats contrastantes. Para el primer muestreo no se anotaron los diferentes hábitats, ya que no se había percibido la importancia de esta variable, por lo que no se llevó a cabo.

La mayor cantidad de lombrices se encontró bajo coberturas vivas de Paspalum conjugatum y bajo la hojarasca de banano en descomposición. Las poblaciones más bajas ocurrieron bajo la cobertura viva de Panicum zizanoides y en suelo desprovisto de vegetación. Para los muestreos cuatro y cinco la población de lombrices bajo Panicum mostró valores intermedios.

Los datos revelan una dinámica más compleja de lo anticipado, pues las especies presentes pueden ser tan importantes como la ausencia de vegetación, para las poblaciones de lombrices.

Cuadro 2. Número de lombrices por $\mathrm{m}^{3}$ bajo hojarasca en descomposición en los diferentes tratamientos de manejo de malezas. Finca San Pablo.

\begin{tabular}{lrrrrrr}
\hline & \multicolumn{6}{c}{ Tratamientos } \\
\cline { 2 - 7 } Muestreo & Testigo & Machete & Glisofato & Paraquat & Glusifonato & Racional \\
\hline $17 / 11 / 00$ & $458 \mathrm{a}$ & $396 \mathrm{a}$ & $375 \mathrm{a}$ & $333 \mathrm{a}$ & $625 \mathrm{a}$ & $812 \mathrm{a}$ \\
$08 / 03 / 01$ & $562 \mathrm{a}$ & $719 \mathrm{a}$ & $531 \mathrm{a}$ & $437 \mathrm{a}$ & $594 \mathrm{a}$ & $552 \mathrm{a}$ \\
$19 / 05 / 01$ & $302 \mathrm{a}$ & $500 \mathrm{a}$ & $333 \mathrm{a}$ & $469 \mathrm{a}$ & $364 \mathrm{a}$ & $500 \mathrm{a}$ \\
$10 / 08 / 01$ & $469 \mathrm{a}$ & $583 \mathrm{a}$ & $396 \mathrm{a}$ & $427 \mathrm{a}$ & $437 \mathrm{a}$ & $594 \mathrm{a}$ \\
$10 / 10 / 01$ & $489 \mathrm{a}$ & $656 \mathrm{a}$ & $656 \mathrm{a}$ & $333 \mathrm{a}$ & $511 \mathrm{a}$ & $792 \mathrm{a}$ \\
\hline
\end{tabular}

Medias con la misma letra de una fila, no difieren según la prueba de DMS protegida de Fisher, al 0,05 de probabilidad 
Cuadro 3. Número de lombrices por $\mathrm{m}^{3}$ bajo cobertura espontánea y bajo hojarasca de banano. Finca San Pablo.

\begin{tabular}{ccc}
\hline Muestreo & $\begin{array}{c}\text { Bajo cobertura } \\
\text { espontánea }\end{array}$ & $\begin{array}{c}\text { Bajo hojaras } \\
\text { de banano }\end{array}$ \\
\hline $17 / 11 / 00$ & $292 \mathrm{a}$ & $500 \mathrm{~b}$ \\
$08 / 03 / 01$ & $361 \mathrm{a}$ & $566 \mathrm{~b}$ \\
$19 / 05 / 01$ & $191 \mathrm{a}$ & $411 \mathrm{~b}$ \\
$10 / 08 / 01$ & $347 \mathrm{a}$ & $484 \mathrm{~b}$ \\
$10 / 10 / 01$ & $361 \mathrm{a}$ & $573 \mathrm{~b}$ \\
\hline
\end{tabular}

Medias con letras distintas para las filas, difieren según la prueba de DMS protegida de Fisher, al 0,05 de probabilidad

Por otra parte, los resultados mostraron una tendencia similar en los cinco muestreos, por lo que se puede decir que la morfología de las malezas presentes, tanto de su parte aérea como de la raíz, juega un importante papel en la conservación de la humedad del suelo y por ende en la cantidad de lombrices bajo cada especie, o en ausencia de ellas.

Durante los primeros tres muestreos se ve un acumulado de lluvias bastante bajo (Cuadro 5), correspondientes a los meses noviembre, marzo y mayo, respectivamente, lo que pudo maximizar diferencias de humedad en el suelo entre los diferentes microhabitats analizados. Aunque no se cuantificó, resultó obvio a la vista una mayor humedad bajo Paspalum conjugatum y hojarasca de banano, que bajo Panicum zizanoides o suelo desprovisto de vegetación.

Cuadro 4. Número de lombrices por $\mathrm{m}^{3}$ bajo diferentes hábitats. Finca San Pablo.

\begin{tabular}{lrrrr}
\hline Muestreo & Paspalum $^{*}$ Hojarasca** $^{*}$ Panicum $^{* * *}$ Sin cobertura \\
\hline $17 / 11 / 00$ & - & - & - & - \\
$08 / 03 / 01$ & $1083 \mathrm{c}$ & $566 \mathrm{~b}$ & $212 \mathrm{a}$ & $241 \mathrm{a}$ \\
$19 / 05 / 01$ & $482 \mathrm{~b}$ & $411 \mathrm{~b}$ & $154 \mathrm{a}$ & $94 \mathrm{a}$ \\
$10 / 08 / 01$ & $549 \mathrm{~b}$ & $484 \mathrm{~b}$ & $419 \mathrm{ab}$ & $231 \mathrm{a}$ \\
$10 / 10 / 01$ & $552 \mathrm{~b}$ & $573 \mathrm{~b}$ & $414 \mathrm{ab}$ & $290 \mathrm{a}$ \\
\hline
\end{tabular}

* Bajo Paspalum el número de muestras fue 6, 7, 9 y 6 para los muestreos del 2 al 5, respectivamente y los datos provienen de las parcelas con tratamientos 1,2 y 6 .

** Bajo hojarasca el número de muestras en cada muestreo fue de 36 y provienen de todas las parcelas.

*** Bajo Panicum el número de muestras fue 10, 13, 7 y 8 para los muestreos del 2 al 5 , respectivamente y provienen de las parcelas con tratamientos $1,2,3,5$ y 6 .

Medias con letras distintas para las filas, difieren según la prueba de DMS protegida de Fisher, al 0,05 de probabilidad
Para los muestreos cuatro y cinco se observa un acumulado mayor, correspondiente a los meses de agosto y octubre, lo que podría explicar el aumento en las poblaciones de lombrices bajo la cobertura de Panicum (Cuadro 4).

En presencia de cobertura y suficiente humedad, tienden a desaparecer las diferencias en poblaciones de lombrices en los primeros $25 \mathrm{~cm}$ del suelo. Cuando la humedad del suelo baja considerablemente, el tipo de vegetación espontánea predominante, puede marcar diferencias en las poblaciones de lombrices, presumiblemente por diferencias en la conservación de humedad en el suelo.

Cuadro 5. Datos de precipitación $(\mathrm{mm})$ en la zona de 28 millas de Limón, Costa Rica en los ocho días previos a los muestreos y el día de los muestreos. Finca San Pablo.

\begin{tabular}{lrrrrr}
\hline & \multicolumn{5}{c}{ Muestreos } \\
\cline { 2 - 6 } Día & $\mathbf{1}$ & $\mathbf{2}$ & $\mathbf{3}$ & $\mathbf{4}$ & $\mathbf{5}$ \\
\hline & 0,0 & 0,8 & 0,0 & 2,0 & 3,2 \\
2 & 8,8 & 1,1 & 13,1 & 9,9 & 0,2 \\
3 & 0,7 & 6,5 & 0,0 & 0,8 & 0,2 \\
4 & 0,0 & 0,1 & 0,0 & 11,1 & 43,9 \\
5 & 0,0 & 0,0 & 0,0 & 27,5 & 17,4 \\
6 & 1,3 & 0,2 & 0,0 & 49,4 & 0,0 \\
7 & 0,0 & 13,7 & 2,5 & 1,6 & 18,6 \\
8 & 14,4 & 0,4 & 17,7 & 3,9 & 0,2 \\
Día del & & & & & \\
muestreo & 15,8 & 0,0 & 0,0 & 4,8 & 0,0 \\
Acumulado & 41 & 22,8 & 33,3 & 111 & 83,7 \\
\hline
\end{tabular}

\section{CONCLUSIONES Y RECOMENDACIONES}

Se puede concluir que a la fecha, las poblaciones de lombrices no se vieron afectadas por algún factor directamente relacionado con los diferentes tratamientos de control de malas hierbas bajo estudio.

El suelo descubierto y la presencia de la maleza Panicum zizanoides como cobertura, mostraron las poblaciones más bajas de lombrices; mientras que la hojarasca de banano en descomposición y Paspalum conjugatum, las poblaciones más altas. El denominador común que se observó para explicar estas diferencias fue la humedad del suelo. 
Si se asume un análisis holístico del agroecosistema, las interacciones se hacen más complejas pues sería de interés determinar si una cobertura como Paspalum conjugatum, si bien favorece la población de lombrices, podría presentar interacciones negativas como hospedera de nemátodos, ratas o culebras, o interferir con el cultivo.

Es de gran interés modelar la dinámica de la hojarasca en el bananal, pues se anticipa que esta, aunada a un manejo racional de malezas, permitirá obtener indicadores de calidad de sitio positivos, incluidas las poblaciones de lombrices.

Con base a los resultados hasta ahora, se anticipa, que conforme los tratamientos promuevan la presencia de vegetación específica, se detectarán diferencias en las poblaciones de lombrices entre éstos, por ese efecto indirecto.

\section{LITERATURA CITADA}

BAUER, C.; RÖMBKE, J. 1997. Factors influencing the toxicity of two pesticides on the three lumbricid species in laboratory test. Soil Biology and Biochemestry 29 (5/6): 705-708.

DAY, L. 1996. The city naturalist - Earthworms (en línea). Consultado 05 julio 2001. Disponible http://www.nysite.com/nature/fauna/earthworm.htm
DONKER, M.; EIJSACKERS, H.; HEIMBACH, F. 1993. Ecotoxicology of soil organisms. USA. Ed. Lewis Publishers. p. 390-393.

EDWARDS, C.A. 1986. Earthworms organic waste and food. Span 26 (3): 106-109.

FRAILE, J. 1986a. Las lombrices de tierra contribuyen al aprovechamiento de los recursos naturales. Biocenosis 2(3-4): 46-50.

FRAILE, J. 1986b. Participación de las lombrices de tierra en el ciclo del nitrógeno, con énfasis en los sistemas agroforestales de Costa Rica. Uniciencia (CR) 3(1-2):119-124.

LEE, K.E, 1985. Earthworms their ecology and relationships with soils and land use. Orlando (Fla). Academic Press. $416 \mathrm{p}$.

LEÓN, S.; GONZÁLEZ, N.; LOBO, M.; FRAILE, J. 1988. Poblaciones de lombrices de tierra en sitios de acumulación de desechos orgánicos en el Valle Central de Costa Rica. Uniciencia (C.R.) 5: 61-68.

POTTER, D.; BUXTON, M.; REDMOND, C.; PATTERSON, C.; POWELL, A. 1990. Toxicity of pesticides to earthworms (Oligochaeta: Lumbricidae) and effect of thatch degradation in Kentucky Bluegrass Turf. Journal of economic entomology 83(6): 2362-2369. 